\title{
Anzahl der gesetzlichen Kassen nimmt ab
}

zent. Laut Studie wird durch die steigenden Zusatzbeiträge die Wechselaktivität der Versicherten deutlich zunehmen. Folglich gebe es vor allem Fusionen zwischen Kassen mit geringen Mitgliederzahlen. Zusätzlich prognostizieren die Befragten durch die demografische Entwicklung und die steigenden Kosten im Gesundheitswesen in spätestens zehn bis 20 Jahren Einschnitte in den gesetzlich vorgegebenen Leistungskatalog der Krankenkassen. Für die Marktstudie hatte die BDO im vergangenen Jahr Vertreter der gesetzlichen und privaten Kassen, Kassenärztlichen Vereinigungen sowie Experten aus Gesundheitspolitik und Sozial- und Wirtschaftswissenschaft per Telefon befragt. Die Studie erfolgte ohne externen Auftrag.

\section{Themen der Mundgesundheit herausragend aufbereitet}

Die Gewinner des Journalistenpreises Abdruck 2016 stehen fest: Andrea Hennis (Focus Gesundheit), das Team von [W] wie Wissen (Das Erste), Stefan Geier (Bayern 2) und Dr. Geraldine Nagel (Onmeda) erhalten den Preis für ihre Beiträge in den Kategorien Print, TV, Hörfunk und Online.

Zum elften Mal in Folge hat die Initiative proDente den Journalistenpreis verliehen. Mit dem „Abdruck“ würdigt sie herausragende journalistische Arbeiten über zahnmedizinische und zahntechnische Themen.

In der Kategorie Print gewann die Autorin Andrea Hennis mit ihrem Artikel „Einfach richtig putzen!“. Der Beitrag liefert eine Vielzahl von wissenswerten Fakten rund um das Zähneputzen und erläutert viele Aspekte mit einer hohen Informationsdichte.

In der Kategorie TV ist die Sendung [W] wie Wissen ganz vorn. Sie ist eine Koproduktion von BR, HR, NDR, SWR und WDR. Eingereicht wurde der Beitrag vom SWR-Team. Die Inhalte stammen vom Bayerischen Rundfunk. Die Folge „Zahn um Zahn Showdown im Mundraum" beleuchtet verschiedene Themen der Mundgesundheit und gibt ein umfassendes Bild von den Mög-

lichkeiten der heutigen und künftigen Zahnmedizin.

„Gute Zähne, gesunder Körper - Zwischen Hightech und Prophylaxe" heißt der ausgezeichnete Radiobeitrag von Stefan Geier. Der Autor gibt einen Überblick über moderne Zahntechnik und zahnmedizinische Therapien und deren Weiterentwicklung zur Erhaltung der Zahngesundheit.

Das schwierige Thema „Molaren-Inzisiven-Hypomineralisation" hat die Autorin Dr. Geraldine Nagel für das Gesundheitsportal Onmeda bearbeitet. Da sie es sehr gut geschafft hat, das „Phänomen“ ausführlich, inhaltlich korrekt und dabei verständlich zu beschreiben, erhielt auch sie den Preis.

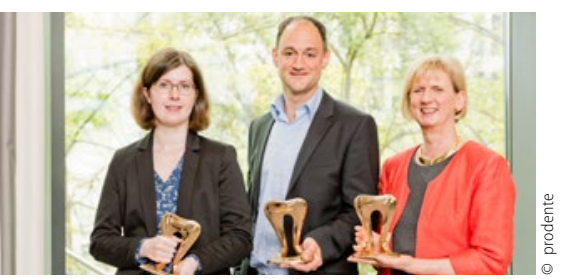

Preisverleihung des Journalistenpreises Abdruck 2016 (von links): Dr. Geraldine Nagel (onmeda), Stefan Geyer (BR), Andrea Hennis (Focus Gesundheit)

\section{Nach den Landtagswahlen}

\section{Spitzen der Gesundheitsressorts stehen fest}

Die neuen Landesregierungen in Baden-Württemberg, SachsenAnhalt und Rheinland-Pfalz haben ihre Verantwortlichen für das Thema „Gesundheit“ bekanntgegeben. In Baden-Württemberg wird mit Manfred Lucha erstmals ein Grünen-Politiker Sozialminister. Lucha ist ausgebildeter Krankenpfleger und studierte Sozialarbeit sowie Management im Sozial- und Gesundheitswesen. Auf dem gesundheitspolitischen Parkett BadenWürttembergs kennt sich Lucha aus. In der vergangenen Legislaturperiode war er Vorsitzender des Arbeitskreises Soziales der Grünen-Landtagsfraktion. Lucha übernimmt das Amt von Katrin Altpeter (SPD).
In Sachsen-Anhalt wird künftig Petra Grimm-Benne (SPD) als Ministerin für Arbeit, Soziales und Integration über die Fragen im Gesundheitsbereich entscheiden. Sie folgt auf Norbert Bischoff (SPD). Die SPD-Politikerin war zuvor Mitglied des Ausschusses für Arbeit und Soziales, Leiterin des Arbeitskreises Soziales der Landtagsfraktion und Sprecherin für Sozial- und Gesundheitspolitik.

Sabine Bätzing-Lichtenthäler (SPD) bleibt Ministerin für Soziales, Arbeit, Gesundheit und Demografie in Rheinland-Pfalz. In diesem Amt ist die SPD-Politikerin seit November 2014. Zwischen 2005 bis 2009 war Bätzing-Lichtenthäler Drogenbeauftragte der Bundesregierung. cas 